\title{
Neurophysiological constraints on the eye-mind link
}

\author{
Erik D. Reichle ${ }^{1 *}$ and Eyal M. Reingold ${ }^{2}$ \\ 'School of Psychology, University of Southampton, Southampton, UK \\ 2 Psychology, University of Toronto, Mississauga, ON, Canada
}

\section{Edited by:}

Merim Bilalic, University Tübingen;

University Clinic, Germany

Reviewed by:

Raymond Bertram, University of

Turku, Finland

Petar Milin, Eberhardt Karls

University Tuebingen, Germany

*Correspondence:

Erik D. Reichle, School of

Psychology, University of

Southampton, Southampton

SO17 1BJ, UK

e-mail: erik.d.reichle@gmail.com
Several current computational models of eye-movement control in reading posit a tight link between the eye and mind, with lexical processing directly triggering most "decisions" about when to start programming a saccade to move the eyes from one word to the next. One potential problem with this theoretical assumption, however, is that it may violate neurophysiological constraints imposed by the time required to encode visual information, complete some amount of lexical processing, and then program a saccade. In this article, we review what has been learned about these timing constraints from studies using ERP and MEG. On the basis of this review, it would appear that the temporal constraints are too severe to permit direct lexical control of eye movements without a significant amount of parafoveal processing (i.e., pre-processing of word $n+1$ from word $n$ ). This conclusion underscores the degree to which the perceptual, cognitive, and motor processes involved in reading must be highly coordinated to support skilled reading, a par excellence example of a task requiring visual-cognitive expertise.

Keywords: ERP, MEG, computational models, reading, saccades

\section{INTRODUCTION}

Reading is one of the most complex tasks that we routinely perform. Part of this complexity reflects the fact that the visual acuity needed to identify the features of printed text seems to be largely limited to a $2^{\circ}$ region of the central visual field, the fovea. Because of this limitation, readers must direct their eyes to the majority of words in a text (Rayner, 1998). And although the eyes normally move through the text so rapidly as to make the "task" of moving the eyes appear effortless, this impression is misleading because individual eye movements, called saccades, require time to program and execute (Becker and Jürgens, 1979) and are subject to both random and systematic motor error (McConkie et al., 1988). For those reasons, the programming and execution of saccades during reading is itself a highly skilled activity. Adding to this complexity is the fact that the "decisions" about when to move the eyes must be coordinated with the other cognitive processes involved in reading, such as the identification of words and the allocation of covert attention. Attempts to better understand these interactions have produced computational models that describe how lexical processing and attention are coordinated with the programming and execution of saccades to produce the patterns of eye movements that are observed with skilled readers (see Reichle et al., 2003).

Although the assumptions of these models are complex and varied, the two most successful of these models, E-Z Reader (Reichle et al., 1998, 2009) and SWIFT (Engbert et al., 2002, 2005), posit that the eyes are tightly coupled to the mind, with moment-to-moment "decisions" about when to move the eyes being controlled by lexical processing. For example, according to E-Z Reader, the completion of a preliminary stage of lexical processing (called the familiarity check) on a word initiates the programming of a saccade to move the eyes to the next word. And according to the SWIFT model, a saccadic program to move the eyes off of a word is initiated by an autonomous (random) timer that can be inhibited if the fixated word is difficult to process. In this way, both models can explain the ubiquitous finding that difficult (e.g., low frequency) words tend to be the recipients of longer fixations than easy (e.g., high frequency) words (Just and Carpenter, 1980; Inhoff and Rayner, 1986; Rayner and Duffy, 1986; Schilling et al., 1998; Rayner et al., 2004; Kliegl et al., 2006).

Because models of eye-movement control have been used to both simulate the "benchmark" findings related to eye movements in reading and examine various theoretical issues related to reading (e.g., how aging affects readers' eye movements; Laubrock et al., 2006; Rayner et al., 2006), the models represent serious attempts to explain the eye-mind link, or interface between lexical processing, on one hand, and eye-movement control, on the other. To the extent that they are successful in this capacity, however, the models raise an important question: How can something as slow as lexical processing mediate the decisions about when to move the eyes? To fully appreciate the paradoxical nature of this question, consider that, although fixation durations are quite variable during reading, occasionally being as short as $50 \mathrm{~ms}$ or as long as $800 \mathrm{~ms}$, most are 200-250 ms in duration (Rayner, 1998). Because of this, and because of the fact that some non-trivial amount of time is required to program a saccade (Becker and Jürgens, 1979), it is not immediately obvious how there can be enough time available during each fixation to allow lexical processing to intervene in the decisions about when to move the eyes.

In the remainder of this article, we will attempt to resolve this paradox by reviewing what has been learned from neurophysiology studies about the time course of those processes that are known to play important functional roles in reading andaccording to models like E-Z Reader and SWIFT-eye-movement 
control during reading. The studies that will be reviewed (see Table 1) employ two basic methods, event-related potentials (ERPs) and megnetoencephalography (MEG), to examine the times required to propagate visual information from the eyes to the brain, and to then visually encode and engage in lexical processing of printed words. It is important to note, however, that although these studies provide estimates of the times required to complete these processes, these estimates are inherently conservative because they correspond to the first statistically reliable effects of, for example, some variable (e.g., word frequency) on ERP markers of lexical processing. We will therefore provide the minimum, mean, and maximum values of each estimate.

\section{RESULTS}

\section{RETINA-BRAIN LAG}

The retina-brain lag is the time required for visual information to propagate from the eyes to the earliest cortical areas of the brain. The duration of this lag has been estimated using ERPs by having subjects attend to a visual stimulus (e.g., checkerboard pattern) that is suddenly displayed on a computer monitor and measuring when a visual-evoked potential (VEP) occurs relative to the onset of the stimulus. Because early cortical areas maintain a retinotopic mapping between spatial locations in the visual field and cortex (Courtney and Ungerleider, 1997), it is possible to localize the neural generators of the VEP and thereby confirm that it reflects early visual processing.

Using this procedure, Clark et al. (1995) found that the VEP had a mean latency of $40-45 \mathrm{~ms}$ post-stimulus onset. Using a similar paradigm but having subjects make recognition decisions about images of faces, George et al. (1997) found that VEPs differentiate previously seen versus novel faces as early as $50 \mathrm{~ms}$ post-stimulus onset, with these repetition effects peaking at around $80 \mathrm{~ms}$. This finding has been replicated (MouchetantRostaing et al., 2000; Seeck et al., 1997), providing additional evidence that $45-50 \mathrm{~ms}$ is sufficient for visual information to reach the brain. Finally, in an experiment designed to examine the time course of both early and late visual processing, Foxe and Simpson (2002) observed a 50-63 ms VEP onset latency when subjects viewed pairs of bilaterally displayed disks with the task of indicating whenever one was displaced from the other. Thus, as Table 1 indicates, estimates of the retina-brain lag range from $47-73 \mathrm{~ms}$ across the studies that were reviewed, with a mean of $60 \mathrm{~ms}$.

Table 1 | Studies (listed chronologically) examining the time course the retina-brain lag, visual encoding, and lexical processing, including their method, task and stimuli, and estimates (in ms) of when the processes occur.

\begin{tabular}{|c|c|c|c|c|}
\hline Process & Study & Method & Task and Stimuli & Mean Estimates [Min, Max] (ms) \\
\hline \multirow[t]{6}{*}{ Retina-Brain Lag } & Clark et al. (1995) & ERP & viewing checkerboard patterns & $42.5[40-45]$ \\
\hline & George et al. (1997) & ERP & recognition of face images & $65[50-80]$ \\
\hline & Seeck et al. (1997) & ERP & recognition of face images & $70[50-90]$ \\
\hline & Mouchetant-Rostaing et al. (2000) & ERP & recognition of face images & $65[45-85]$ \\
\hline & Foxe and Simpson (2002) & ERP & detecting displaced visual disks & $57.5[50-63]$ \\
\hline & \multicolumn{3}{|c|}{ Mean Estimates } & 60 [47-73] \\
\hline \multirow[t]{7}{*}{ Visual Encoding } & Van Rullen and Thorpe (2001) & ERP & categorizing vehicles vs. animals & $77.5[75-80]$ \\
\hline & Foxe and Simpson (2002) & ERP & detecting displaced visual disks & $77.5[70-85]$ \\
\hline & Assadollahi and Pulvermüller (2001) & MEG & detecting novel words & $105[90-120]$ \\
\hline & Assadollahi and Pulvermüller (2003) & MEG & detecting novel words & $90[60-120]$ \\
\hline & Hauk and Pulvermüller (2004) & ERP & lexical decision of letter strings & $102.5[80-125]$ \\
\hline & Hauk et al. (2006) & ERP & lexical decision of letter strings & $95[90-100]$ \\
\hline & \multicolumn{3}{|c|}{ Mean Estimates } & $91.3[77.5-105]$ \\
\hline \multirow[t]{11}{*}{ Lexical Processing } & Sereno et al. (1998) & ERP & lexical decision of letter strings & $148[132-164]$ \\
\hline & Assadollahi and Pulvermüller (2001) & MEG & detecting novel words & $145[120-170]$ \\
\hline & Assadollahi and Pulvermüller (2003) & MEG & detecting novel words & $145[120-170]$ \\
\hline & Sereno et al. (2003) & ERP & word-by-word reading & 162 [132-192] \\
\hline & Hauk and Pulvermüller (2004) & ERP & lexical decision of letter strings & $170[150-190]$ \\
\hline & Proverbio et al. (2004) & ERP & phoneme detection in words & 155 [135-175] \\
\hline & Baccino and Manunta (2005) & ERP & semantic relatedness judgment & 167 [119-215] \\
\hline & Dambacher et al. (2006) & ERP & natural sentence reading & $170[140-200]$ \\
\hline & Hauk et al. (2006) & ERP & lexical decision of letter strings & $110[100-120]$ \\
\hline & Penolazzi et al. (2007) & ERP & word-by-word reading & 120 [110-130] \\
\hline & Reichle et al. (2011) & ERP & lexical decision of letter strings & 132 [102-162] \\
\hline
\end{tabular}




\section{VISUAL ENCODING}

As with the retina-brain lag, the minimal time required to engage in visual encoding has been estimated by examining when differential effects related to the visual properties of stimuli that are suddenly displayed on a computer monitor are first discernable in the ERP record. For example, Van Rullen and Thorpe (2001) had subjects make categorization decisions about photographs of vehicles versus animals and found category-related differences in ERP components as early as $75-80 \mathrm{~ms}$ post-stimulus onset. Similarly, Foxe and Simpson (2002) found ERP components that could be localized to the infero-temporal, parietal, and dorsolateral-prefrontal regions (which have been implicated in high-level visual processing; Van Essen and DeYoe, 1995) were active by $70-85 \mathrm{~ms}$ post-stimulus onset, suggesting that these higher-level visual-processing regions can modulate visual processing in earlier regions via feedback in as little as $30 \mathrm{~ms}$ after processing begins in those earlier regions.

Similar estimates of the time course of visual encoding have also been reported in tasks involving (more global aspects of) lexical processing. For example, Hauk and Pulvermüller (2004) observed effects of word length on ERP components after 80-125 ms when subjects made lexical decisions about short and long letter strings. And using a similar methodology, Hauk et al. (2006) observed word-length effects within 90-100 ms. Two other studies (Assadollahi and Pulvermüller, 2001, 2003) demonstrated the generality of these results by examining the time course of visual encoding of printed words using MEG. In these studies, subjects first memorized a list of short and long high- and lowfrequency words and then viewed a random sequence comprised of those words and new words with instructions to press a button whenever they saw a new word. The key finding related to visual encoding were effects of word length, which were evident after 90-120 ms in the first study and after 60-120 ms in the second, indicating that visual properties of the words (i.e., their length) are encoded in as little as $10-40 \mathrm{~ms}$ after visual information had been propagated from the eyes to the brain. Thus, as Table 1 shows, the studies reviewed in this section collectively suggest that visual encoding occurs within $77.5-105 \mathrm{~ms}$, with a mean of $91.3 \mathrm{~ms}$.

\section{LEXICAL PROCESSING}

For the purposes of this review, lexical processing will refer to mental operations that convert the visual representation of a word into its (abstract) orthographic form so that that information can be used to access that word's pronunciation and/or meaning. Unfortunately, attempts to determine the time course of lexical processing using neurophysiological methods have produced somewhat inconsistent results. For example, Sereno et al. (1998) conducted a seminal ERP experiment in which subjects made lexical decisions about letter strings that included high- and low-frequency words. (Because the frequency with which a word is encountered in text affects how rapidly its form and meaning can be accessed from memory, word-frequency effects are indicators that lexical processing is well underway; Hudson and Bergman, 1985) Sereno et al. found that word frequency modulated ERP components after only 132-164 ms, suggesting that lexical processing occurs very rapidly. Similarly,
Hauk et al. (2006) observed word-frequency effects even earlier, by $100-120 \mathrm{~ms}$.

This conclusion was bolstered by similar findings using both ERP and MEG. For example, the two MEG studies mentioned earlier (in relation to visual encoding) found a word-frequency effect (Assadollahi and Pulvermüller, 2001) and interaction between word frequency and length (Assadollahi and Pulvermüller, 2003) after 120-170 ms. Early lexical effects have also been observed in ERP experiments in which subjects read sentences that were displayed one word at a time: Penolazzi et al. (2007) observed a Frequency $\times$ Length interaction after $110-130 \mathrm{~ms}$, and Sereno et al. (2003) observed a frequency effect after 132-192 ms. And similarly, Proverbio et al. (2004) observed word-frequency effects after 135-175 ms in an ERP experiment in which subjects detected target phonemes embedded in visually displayed words.

This evidence for rapid lexical processing must, however, be reconciled with results suggesting that such processing can be much less rapid. For example, Hauk and Pulvermüller (2004) had subjects make lexical decisions about letter strings that contained short and long high- and low-frequency words and found that ERP components were modulated by frequency after 150-190 ms. Similarly, Dambacher et al. (2006) recorded ERPs from subjects who read sentences and found word-frequency effects after 140-200 ms. Consequently, an important challenge for future investigations of the time course of lexical processing would be to isolate the methodological differences that produced the mixed pattern of results in the literature.

Because our goal is to better characterize the relationship between lexical processing and saccadic programming, the final two studies that will be reviewed are particularly important because they were explicitly designed to examine this relationship using ERP. In the first, Baccino and Manunta (2005) had subjects move their eyes to two peripherally-displayed words to make semantic-related judgments about those words. The key finding was that the frequency of the second word modulated ERP components after only 119-215 ms when the data were timelocked to the fixation on the first word, which was interpreted as evidence for rapid parafoveal lexical processing of the second word. In the second study, Reichle et al. (2011) had subjects move their eyes from centrally- to peripherally-displayed letter strings to indicate whether either was a non-word. The key finding was that the frequency of the central word modulated ERP components after only 102-162 ms when the data were time-locked to the onset of the saccade to the peripheral word, which was interpreted as evidence that an early stage of lexical processing initiates saccadic programming. These results, in combination with those of the other studies reviewed in this section, suggest that lexical processing is well under way by $126.6-171.8 \mathrm{~ms}$, with a mean of $147.6 \mathrm{~ms}$. However, it is important to note that, with the exception of Dambacher et al.'s (2006) experiment, these estimates were obtained using non-reading tasks that preclude normal parafoveal processing of upcoming words.

\section{DISCUSSION}

To better understand the theoretical implications of this review, it is instructive to superimpose the estimated process durations 
(Table 1) on a time-line corresponding to the amount of time available for cognitive processing during a single fixation of reading. Figure 1A thus shows the time course of the retina-brain lag, visual encoding, and lexical processing are aligned to the onset of a single 240-ms fixation. (Although this particular duration is arbitrary and ignores the issue of variability, it corresponds to the mean single-fixation duration observed on low-frequency words and is therefore a conservative estimate of the time available for lexical processing during most fixations; Reingold et al., 2012.)

As Figure 1A shows, the neurophysiological estimates suggest that, on average, $148 \mathrm{~ms}$ is required to visually encode a printed word and then complete some amount of lexical processing of that word. However, because the fixation is only $240 \mathrm{~ms}$ in duration, there is seemingly little time to complete all of the operations that are necessary to move the eyes off of the word $92 \mathrm{~ms}$ later. These operations (at a minimum) include the transmission of a signal to the oculomotor system to start programming a saccade, the actual programming of that saccade, and whatever afferent delay occurs in the brainstem circuitry prior to moving the eyes. The conclusion that so little time is available to complete these operations is seemingly at odds with eye-movement experiments suggesting that saccades require $125-200 \mathrm{~ms}$ to program

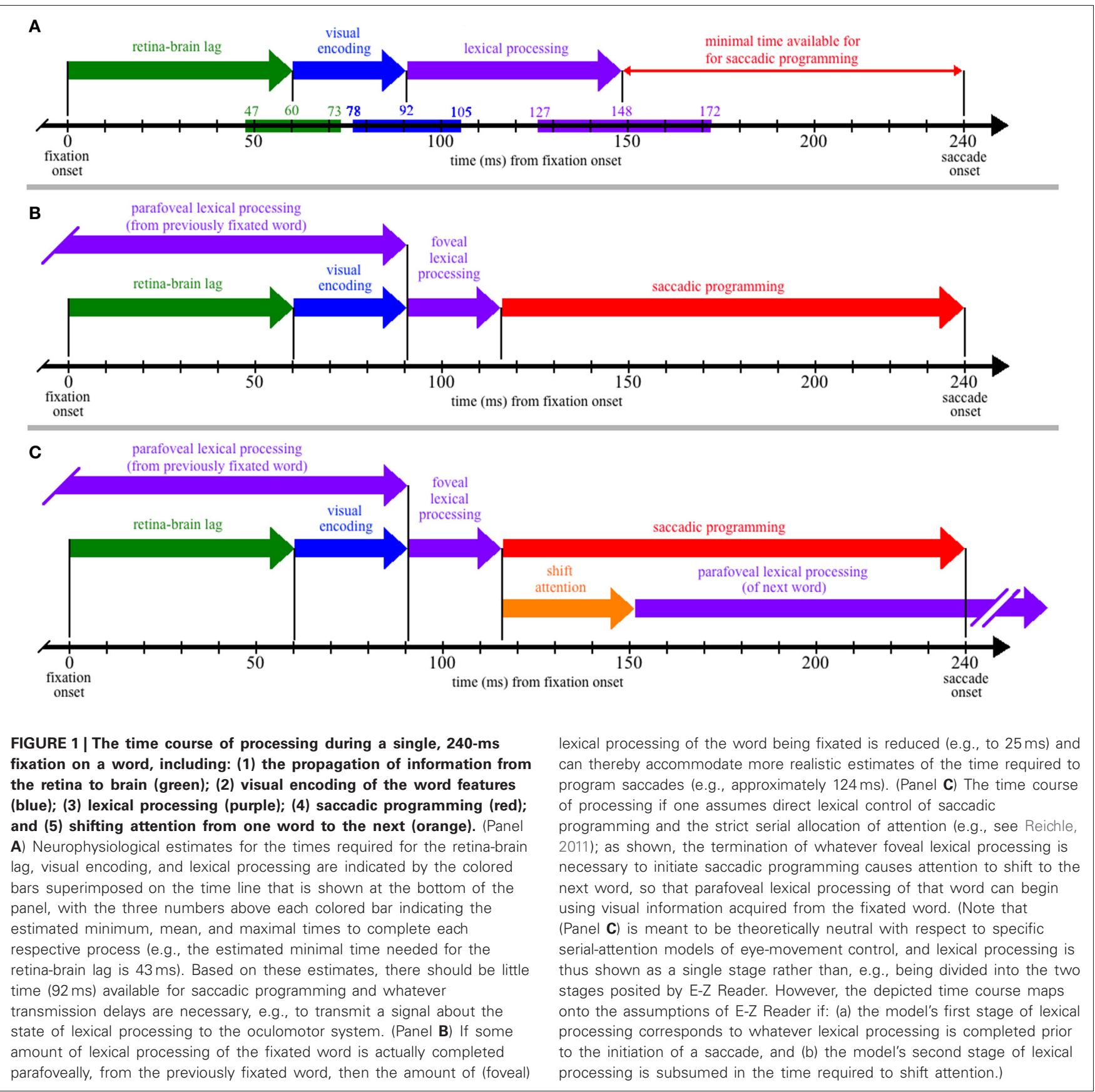


(Becker and Jürgens, 1979; Rayner et al., 1983; Reingold et al., 2012). It is also at odds with models of eye-movement control in reading, which posit that lexical processing is the "engine" that cause the eyes to progress through the text (Reichle et al., 1998, 2009; Engbert et al., 2002, 2005). Our analysis of the time course of lexical processing thus poses a paradox if one is to maintain the position that the completion of some amount of lexical processing is what determines when the eyes move during reading.

The solution to this paradox is that a significant portion of the lexical processing of a word that must be completed to "trigger" saccadic programming is actually completed from the preceding word, using visual information that was acquired from the parafovea. How this happens is illustrated in Figure 1B, which is similar to Figure 1A except that lexical processing of the currently fixated word begins from the previously fixated word, so that only $25 \mathrm{~ms}$ of lexical processing of the fixated word is actually completed from that word. (Again, this precise value is arbitrary, ignores variability, and is only meant to provide an example.) Under this assumption, there is ample time ( $\sim 124 \mathrm{~ms})$ for whatever neural transmission is required to signal the oculomotor system to program and then initiate a saccade. This hypothesis about the importance of parafoveal processing is consistent with survival analyses of fixation durations on high- and low-frequency words with versus without parafoveal preview: Word-frequency effects were discernable more than $110 \mathrm{~ms}$ earlier with than without preview (Reingold et al., 2012).

Finally, to make this hypothesis more concrete, Figure 1C shows the typical sequence of events that are posited to occur by eye-movement models in which attention is allocated to support the lexical processing of exactly one word at any given time (e.g., E-Z Reader or EMMA; see Reichle, 2011). As shown, the lexical processing of any given word is completed from two locationsfrom the previously fixated word and from a fixation on the word itself. Then, upon completing whatever lexical processing is necessary to initiate saccadic programming, attention shifts to the

\section{REFERENCES}

Assadollahi, R., and Pulvermüller, F. (2001). Neuromagnetic evidence for early access to cognitive representations. Neuroreport 12, 207-213. doi: 10.1097/00001756-20010212000007

Assadollahi, R., and Pulvermüller, F. (2003). Early influences of word length and frequency: a group study using MEG. Neuroreport 14, 1183-1187. doi: 10.1097/00001756200306110-00016

Baccino, T., and Manunta, Y. (2005). Eye-fixation-related potentials: insights into parafoveal processing. J. Psychophysiol. 19, 204-215. doi: 10.1027/0269-8803.19.3.204

Becker, W., and Jürgens, R. (1979). An analysis of the saccadic system by means of double step stimuli. Vis. Res. 19, 967-983. doi: 10.1016/00426989(79)90222-0

Clark, V. P., Fan, S., and Hillyard, S. A. (1995). Identification of early

next word so that lexical processing of that word can begin using information acquired from the current fixation location. (Note that, according to the E- $\mathrm{Z}$ Reader model, the time required to shift attention also includes whatever additional time is needed to complete lexical access of the fixated word.)

Of course, the manner in which E-Z Reader instantiates eyemovement control places the most severe temporal constraints on lexical processing and its coordination with the oculomotor system because only one word is processed at a time. These constraints are significantly relaxed to the extent that multiple words are processed in parallel, as posited by the SWIFT model (Engbert et al., 2002, 2005). According to this alternative theoretical perspective, difficulty associated with processing the fixated word can inhibit the autonomous timer that otherwise initiates a saccadic program to move the eyes to a new viewing location. Recent studies on saccadic inhibition (Reingold and Stampe, 1999, 2000, 2002, 2003, 2004) and prior neurophysiological findings (Munoz et al., 1996) suggest that this type of hypothesized mechanism might produce a very rapid inhibitory effect in as little as $20-30 \mathrm{~ms}$. Consequently, there seems to be ample time for an inhibitory mechanism to intervene in the decisions about when to move the eyes during reading (e.g., see Reingold et al., 2012 for a proposal of a hybrid eye-movement control mechanism incorporating both facilitatory and inhibitory lexical influences).

This review thus indicates that lexical processing is sufficiently rapid to permit direct control of the decisions about when to move the eyes during reading, but that such control also requires a substantial amount of lexical processing from the parafoveaperhaps more that has been acknowledged by reading researchers. This latter conclusion underscores the more basic claim about eye-movement control during reading being a highly skilled activity —one that requires a tremendous degree of coordination between the systems that support attention, word identification, and the programming and execution of saccades.

Engbert, R., Nuthmann, A., Richter, E., and Kliegl, R. (2005). SWIFT: a dynamical model of saccade generation during reading. Psychol. Rev. 112, 777-813. doi: 10.1037/0033295X.112.4.777

Foxe, J. J., and Simpson, G. V. (2002). Flow of activation from $\mathrm{V} 1$ to frontal cortex in humans: a framework for defining "early" visual processing. Exp. Brain Res. 142, 139-150. doi: 10.1007/s00221-0010906-7

George, N., Jemel, B., Fiori, N., and Renault, B. (1997). Face and shape repetition effects in humans: a spatio-temporal ERP study. Neuroreport 8, 1417-1423. doi: 10.1097/00001756-19970414000019

Hauk, O., Davis, M. H., Ford, M., Pulvermüller, F., and MarslenWilson, W. D. (2006). The time course of visual word recognition as revealed by linear analysis of ERP data. Neuroimage 30, 1383-1400. doi: 10.1016/j.neuroimage.2005.11.048

Hauk, O., and Pulvermüller, F. (2004). Effects of word length and frequency on the human event-related potential. Clin. Neurophysiol. 115, 1090-1103. doi: 10.1016/j.clinph.2003.12.020

Hudson, P. T., and Bergman, M. W. (1985). Lexical knowledge in word recognition: word length and word frequency in naming and lexical decision tasks. J. Mem. Lang. 24, 46-58. doi: 10.1016/0749-596X(85)90015-4

Inhoff, A. W., and Rayner, K. (1986). Parafoveal word processing during eye fixations in reading: effects of word frequency. Percept. Psychophys. 40, 431-439. doi: 10.3758/BF03208203

Just, M. A., and Carpenter, P. A. (1980). A theory of reading: om eye fixations to comprehension. 
Psychol. Rev. 87, 329-354. doi: 10.1037/0033-295X.87.4.329

Kliegl, R., Nuthmann, A., and Engbert, R. (2006). Tracking the mind during reading: the influence of past, present, and future words on fixation durations. J. Exp. Psychol. Gen. 135, 12-35. doi: 10.1037/00963445.135.1.12

Laubrock, J., Kliegl, R., and Engbert, R. (2006). SWIFT explorations of age differences in eye movements during reading. Neurosci. Behav. Rev. 30, 872-884. doi: 10.1016/j.neubiorev.2006.06.013

McConkie, G. W., Kerr, P. W., Reddix, M. D., and Zola, D. (1988). Eye movement control during reading: I. The location of initial fixations on words. Vis. Res. 28, 1107-1118. doi: 10.1016/0042-6989(88)90137-X

Mouchetant-Rostaing, Y., Giard, M.-H., Bentin, S., Aguera, P.-E., and Pernier, J. (2000). Neurophysiological correlates of face gender processing in humans. Eur. J. Neurosci. 12, 303-310. doi: 10.1046/j.1460-9568.2000.00888.x

Munoz, D. P., Waitzman, D. M., and Wurtz, R. H. (1996). Activity of neurons in monkey superior colliculus during interrupted saccades. J. Neurophysiol. 75, 2562-2580.

Penolazzi, B., Hauk, O., and Pulvermüller, F. (2007). Early semantic context integration and lexical access as revealed by event-related brain potentials. Biol. Psychol. 74, 374-388. doi: 10.1016/j.biopsycho.2006.09.008

Proverbio, A. M., Vecchi, L., and Zani, A. (2004). From orthography to phonetics: ERP measures of grapheme-to-phoneme conversion mechanisms in reading. J. Cogn. Neurosci. 16, 301-317. doi: 10.1162/089892904322984580

Rayner, K. (1998). Eye movements in reading and information processing: 20 years of research. Psychol. Bull. 124, 372-422. doi: 10.1037/0033-2909.124.3.372

Rayner, K., Ashby, J., Pollatsek, A., and Reichle, E. D. (2004). The effects of frequency and predictability on eye fixations in reading: implications for the $\mathrm{E}-\mathrm{Z}$ Reader model. J. Exp. Psychol. Hum. Percept. Perform. 30, 720-732. doi: 10.1037/0096-1523.30.4.720

Rayner, K., and Duffy, S. A. (1986). Lexical complexity and fixation times in reading: effects of word frequency, verb complexity, and lexical ambiguity. Mem. Cogn. 14, 191-201. doi: 10.3758/BF03197692

Rayner, K., Reichle, E. D., Stroud, M. J., Williams, C. C., and Pollatsek, A. (2006). The effects of word frequency, word predictability, and font difficulty on the eye movements of young and older readers. Psychol. Aging 21, 448-465. doi: 10.1037/0882-7974.21.3.448

Rayner, K., Slowiaczek, M. L., Clifton, C., and Bertera, J. H. (1983). Latency of sequential eye movements: implications for reading. J. Exp. Psychol. Hum. Percept. Perform. 9, 912-922. doi: 10.1037/0096-1523.9.6.912

Reichle, E. D. (2011). "Serial attention models of reading," in Oxford Handbook on Eye Movements, eds S. P. Liversedge, I. D. Gilchrist, and S. Everling (Oxford: Oxford University Press), 767-786.

Reichle, E. D., Pollatsek, A., Fisher, D. L., and Rayner, K. (1998). Toward a model of eye movement control in reading. Psychol. Rev. 105, 125-157. doi: 10.1037/0033-295X. 105.1 .125

Reichle, E. D., Rayner, K., and Pollatsek, A. (2003). The E-Z Reader model of eye movement control in reading: comparisons to other models. Behav. Brain Sci. 26, 445-476. doi: 10.1017/S0140525X03000104

Reichle, E. D., Tokowicz, N., Liu, Y., and Perfetti, C. A. (2011). Testing and assumption of the E$\mathrm{Z}$ Reader model of eye-movement control in reading: using eventrelated potentials to examine the familiarity check. Psychophysiology 48, 993-1003. doi: 10.1111/j.14698986.2011.01169.x

Reichle, E. D., Warren, T., and McConnell, K. (2009). Using
E-Z Reader to model the effects of higher-level language processing on eye movements during reading. Psychon. Bull. Rev. 16, 1-21. doi: 10.3758/PBR.16.1.1

Reingold, E. M., Reichle, E. D., Glaholt, M. G., and Sheridan, H. (2012). Direct lexical control of eye movements in reading: evidence from survival analysis of fixation durations. Cogn. Psychology 65, 177-206. doi: 10.1016/j.cogpsych.2012.03.001

Reingold, E. M., and Stampe, D. M. (1999). "Saccadic inhibition in complex visual tasks," in Current Oculomotor Research: Physiological and Psychological Aspects, eds W. B. Cer, H. Deubel, and T. Mergner (London: Plenum), 249-255.

Reingold, E. M., and Stampe, D. M (2000). "Saccadic inhibition and gaze contingent research programs," in Reading as a Perceptual Process, eds A. Kennedy, R. Radach, D. Heller, and J. Pynte (Amsterdam, Holland: Elsevier), 119-145.

Reingold, E. M., and Stampe, D. M. (2002). Saccadic inhibition in voluntary and reflexive saccades. J. Cogn. Neurosci. 14, 371-388. doi: $10.1162 / 089892902317361903$

Reingold, E. M., and Stampe, D. M. (2003). "Using the saccadic inhibition paradigm to investigate saccadic control in reading," in The mind's Eye: Cognitive and Applied Aspects of Eye Movement Research, eds J. Hyönä, R. Radach, and H. Deubel (Amsterdam, Holland: Elsevier), 347-360.

Reingold, E. M., and Stampe, D. M. (2004). Saccadic inhibition in reading. J. Exp. Psychol. Hum. Percept. Perform. 30, 194-211. doi: 10.1037/0096-1523.30.1.194

Seeck, M., Michel, C. M., Mainwaring, N., Cosgrove, R., Blume, H., Ives, J., et al. (1997). Evidence for rapid face recognition from human scalp and intracranial electrodes. Neuroreport 8, 2749-2754. doi: 10.1097/00001756-19970818000021

Sereno, S. C., Brewer, C. C., and O’Donnell, P. J. (2003). Context effects in word recognition: evidence for early interactive processing. Psychol. Sci. 14, 328-333.

Sereno, S. C., Rayner, K., and Posner, M. I. (1998). Establishing a timeline of word recognition: evidence from eye movements and eventrelated potentials. Neuroreport 9, 2195-2200. doi: 10.1097/00001756199807130-00009

Schilling, H. E. H., Rayner, K., and Chumbley, J. I. (1998). Comparing naming, lexical decision, and eye fixation times: word frequency effects and individual differences. Mem. Cogn. 26, 1270-1281. doi: 10.3758/BF03201199

Van Essen, D. C., and DeYoe, E. A. (1995). "Concurrent processing in the primate visual cortex," in The Cognitive Neurosciences, ed M. S. Gazzaniga (Cambridge, MA: MIT Press), 383-400.

Van Rullen, R., and Thorpe, S. J. (2001). The time course of visual processing: from early perception to decision-making. J. Cogn. Neurosci. 13, 454-461. doi: 10.1162/08989 290152001880

Conflict of Interest Statement: The authors declare that the research was conducted in the absence of any commercial or financial relationships that could be construed as a potential conflict of interest.

Received: 01 May 2013; paper pending published: 28 May 2013; accepted: 23 June 2013; published online: 15 July 2013

Citation: Reichle ED and Reingold EM (2013) Neurophysiological constraints on the eye-mind link. Front. Hum. Neurosci. 7:361. doi: 10.3389/fnhum.2013.00361 Copyright (C) 2013 Reichle and Reingold. This is an open-access article distributed under the terms of the Creative Commons Attribution License, which permits use, distribution and reproduction in other forums, provided the original authors and source are credited and subject to any copyright notices concerning any third-party graphics etc. 